\title{
Digital Image Watermarking in Robust Feature Region Set
}

\author{
Seema \\ Malshe(Gondhalekar)
}

\author{
Hitesh Gupta
}

\begin{abstract}
Digital image watermarking is widely used for copyright protection of digital information. The effectiveness of a digital watermarking technique is indicated by the robustness of embedded watermarks against various attacks. A new method for watermarking is suggested is feature based watermarking. For getting robust watermark, the watermark should be embedded in silent part of the data and for these significant features of data is used. In this paper few methods of feature extraction as Harris Laplacian, Laplacian-of-Gaussian, Susan, Gilles are applied for feature extraction. Robust Non overlapping regions against different attacks are selected for watermarking. Comparison for robust feature selection is done against different feature extraction methods. In next stage those regions are pruned to get minimal primary feature region set using pruning algorithm and watermark is embedded in selected regions and then again results of extracted watermark is compared against different feature selection methods for robustness.
\end{abstract}

\section{General Terms}

Watermarking

\section{Keywords}

Robust, Attacks, features, feature extraction, Corner

\section{INTRODUCTION}

There is extensive use of digital multimedia data over the network. It creates a new demand for copyright protection of digital data. Encryption and digital watermarking are the techniques used for copyright protection[1]. Watermarking is embedding a secret signal (a watermark) into the original data, which are always remains present.

The important aspects for the design of watermarking systems are Imperceptibility, Robustness, Security and Capacity. Robustness means the watermark must be robust to common signal processing manipulations and attempts to remove or impair the watermark.

Robustness against attacks[2,3] is an important aspect for watermarking schemes.

\subsection{Attacks}

The wide class of existing attacks can be divided into four main groups removal attacks, geometrical attacks, cryptographic attacks and protocol attacks.

Removal attacks :Removal attacks attempt to weaken or completely remove a watermark from its associated content, while preserving the content so that it is not useless after the attack is over. This category includes Mean/median/Gaussian filtering, Wiener-Lee filtering, Averaging $\mathrm{N}$ instances of the same image, bearing different watermarks. Lossy compression (JPEG, JPEG2000).Sharpening, Contrast enhancement (histogram equalization),Gamma correction, Color quantization/sub sampling Additive/multiplicative noise Gaussian, uniform, or salt-pepper, Multiple watermarking etc
Geometric attacks: Geometric distortions are specific to images including operations as rotation, scaling, translation, cropping etc Cryptographic attacks: Cryptographic attacks aim at cracking the security methods in watermarking schemes and thus finding a way to remove the embedded watermark information or to embed misleading watermarks. Protocol attacks: One type of protocol attack is based on the concept of invertible watermarks. The idea behind inversion is that the attacker subtracts his own watermark from the watermarked data and claims to be the owner of the watermarked data. Another protocol attack is the copy attack. In this case, the goal is not to destroy the watermark or impair its detection, but to estimate a watermark from watermarked data and copy it to some other data, called target data.

Robustness Testing can be done using StirMark [4] which is a generic tool for simple robustness testing of image watermarking algorithms. PSNR and Normalized Correlation of Watermarked Image should be checked for different attacks. PSNR (Peak signal to Noise Ratio) is used to measure the quality of watermarked image., Watermarked Image quality is better if PSNR is bigger. PSNR for image with size $\mathrm{Mx} \mathrm{N}$ is given by:

$$
P S N R=10 * \log _{10}\left(\frac{255}{\sqrt{\frac{1}{M N} \sum_{y=1}^{M} \sum_{x=1}^{N}\left[I(x, y)-I^{\prime}(x, y)\right]^{2}}}\right)
$$

where $\mathrm{I}(\mathrm{x}, \mathrm{y})$ is the original image, $\mathrm{I}^{\prime}(\mathrm{x}, \mathrm{y})$ is watermarked image and $\mathrm{M}, \mathrm{N}$ are the dimensions of the images.

In this paper we are focusing on robustness that is resistant to attack such as filtering, additive noise, compression, RST and other forms of image manipulation, which is very important property of watermark.

\subsection{Digital watermarking techniques:}

There are two main categories of digital watermarking techniques, which are based on the embedding position, spatial domain and frequency domain watermark and other new technique is feature based watermarking.

i) Spatial domain techniques: In this technique, the values at the image pixels are directly modified using on the watermark which is to be embedded. The last significant bits (LSB) technique: One of the most earliest technique. It is implemented by modifying the last significant bits (LSB) of the image's pixel data.

ii) Frequency domain : In this technique the transform coefficients are modified instead of directly changing the pixel values. To detect watermark, the inverse transform is used. The transforms commonly used for watermarking purposes are the discrete cosine transforms (DCT)[5,6], discrete Fourier transforms (DFT) and discrete wavelet transforms (DWT).

iii) Feature based watermarking : A feature is meaningful characteristics of data. Features of an image can are edge, cornet, texture, color etc. 
As features of the image have high invariance to distortions, they can be used as a key to find the insertion location. Watermarking algorithms using a feature of an image were proposed as the second generation watermark $[7,8,9]$ Feature detector methods are classified according to their invariance to rotation or similarity or affine and perspective. The goal is to resist both geometric distortion and signal processing attacks.

The basic three processes involved in watermarking are watermark embedding, applying attacks, watermark detection. In this paper we suggest that before watermark embedding there is step as feature extraction and the watermark is embedded in in silent part of the data. In watermark embedding, a watermark signal is constructed and then embedded into an original image to produce the watermarked image. After embedding is done, the watermarked image can be subjected to various attacks. During watermark detection, the watermark detector is given a test signal that may be watermarked, attacked or not. The watermark detector reports whether the watermark is present or not on examining the signal at its input.

\section{FEATURE-BASED WATERMARKING}

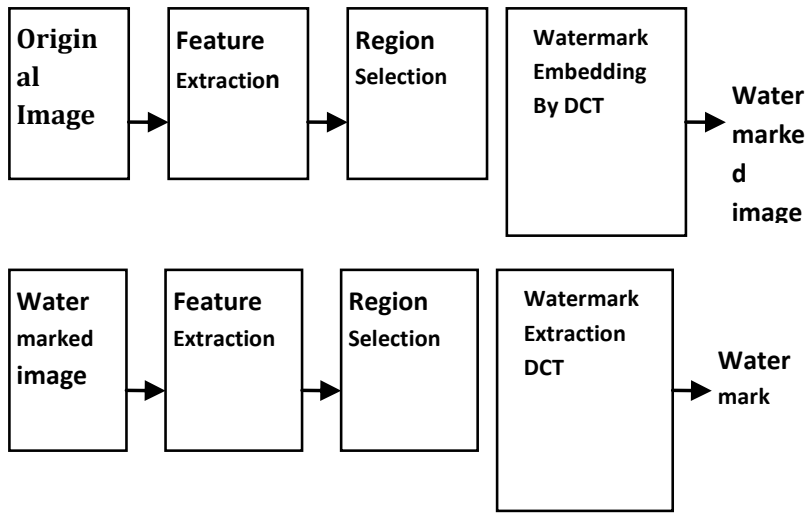

Fig 2.1. Block diagram of feature based watermark Embedding and extraction

The proposed technique is based on feature based watermarking. For getting robust watermark, the watermark should be embedded in silent part of the data. For these significant features of data is used. A feature is meaningful characteristics of data. Features of an image are edge, cornet, texture, color, blob etc. A corner is important localized twodimensional image structure. It has "T", "Y" and "X" junctions. Corners are important points in images which can be easily calculated using mathematics. In computer vision image corner detection is an important task, because corners are proven to be stable across sequences of image.

Features should have some properties for watermarking as resistant to transformations such as rotation, scaling, translation (RST), resistant to noise and localized. An invariant feature is a feature calculated from an image that is invariant with respect to certain transformations, i.e. it does not change when these transformations are applied to the image. The transformations considered here are mainly translation, rotation, and scaling.

In general, feature-based watermark algorithms are the best approaches to resisting geometric distortions since feature points provide stable references for both watermark embedding and detection.
Literature suggests different feature extraction techniques as In paper[7] they develop a robust watermarking scheme using Mexican Hat wavelet scale interaction.. Feature extraction method adopted is it allows different degrees of robustness (against distortion) by choosing proper scale parameters. In paper [8], comparison of technique used in [7] and [8] is compared. They suggest that, the feature detector is a key role in the feature-based watermarking. Up to present, many feature detectors have been proposed such as Hessian-Affine detector, Harris Laplacian etc.

In this paper we compared few different techniques as Harris Laplacian[12,13], LoG[10,12,13], Susan[10,13,14] and Gilles[11] feature extraction methods and then compared all by applying different attacks on regions and Feature survival after attacks is checked.

\section{FEATURE EXTRACTION}

Since a robust feature region for resisting various attacks can be obtained by a good feature detector, the feature detector is a key role in the feature-based watermarking. Up to present, feature detectors have been proposed such as Hessian-Affine detector, MSER, IBR etc. Few other feature extraction techniques are as follows.

\subsection{Laplacian-of-Gaussian (LoG) [12,13]}

The Laplacian of Gaussian method was invented by Marr and Hildrethw in 1980. This method is combination of Gaussian filtering with the use of Laplacian operator. The most common blob detectors are based on the Laplacian of the Gaussian (LoG). For a scale selection filter, the (scalenormalized) Laplacian-of-Gaussian (LoG) is a popular choice. The Laplacian of an image preferred for edge detection because it detects regions of rapid intensity change. The operator of LoG calculates the second spatial derivative of an image. When there is constant intensity in then image areas, then the LoG response will be zero. At the portion of an image where a change in intensity exists, we find positive LoG response on darker sides and negative response at lighter sides. Its 2D filter mask takes the shape of a circular center region with positive weights, surrounded by another circular region with negative weights. The filter response is therefore strongest for circular image structures whose radius corresponds to the filter scale.

1) The Laplacian $L(x, y)$ of an image with pixel intensity values $I(x, y)$ calculated using following equation

$$
L(x, y)=\frac{\partial^{2} I}{\partial x^{2}}+\frac{\partial^{2} I}{\partial y^{2}}
$$

The Laplacian can be calculated using standard convolution methods. Using kernel as follows,.

$$
\begin{array}{ccc}
0 & -1 & 0 \\
-1 & 4 & -1 \\
0 & -1 & 0
\end{array}
$$

2) There is preprocessing step for an image is done, because these kernels are approximating a second derivative measurement on the image, are very sensitive to noise. Gaussian smoothing is applied before applying the Laplacian filter to reduce noise compnent. This pre-processing step reduces the high frequency noise components prior to the differentiation step.

3) We apply the Gaussian smoothing filter with the Laplacian filter first of all, and then convolve this hybrid filter with the image to achieve the required result

Based on the idea of Scale Invariant Region Detection, Lindeberg introduced a detector for blob-like features that searches for scale space extrema of a scale normalized Laplacian-of-Gaussian 
Lindeberg proposed a detector for blob-like features that searches for scale space extrema of a scale-normalized Laplacian-of-Gaussian (LoG).

$$
\mathrm{L}(\mathrm{x}, \sigma)=\sigma^{2}\left(\mathrm{I}_{\mathrm{xx}}(\mathrm{x}, \sigma)+\mathrm{I}_{\mathrm{yy}}(\mathrm{x}, \sigma)\right)
$$

In Laplacian of Gaussian detection, there are mainly three steps

- Filtering

- Enhancement

- Detection

Advantages:

- Find correct places of edges.

- Testing wider area other image. Disadvantages:

- It Malfunction at corners, curves and where the gray level intensity function varies.

- Because of using the Laplacian filter, it is not finding the orientation of edge

\section{2 The Harris-Laplacian Detector [12,13]}

This is based on the idea of auto-correlation. The Harris Laplacian detector relies heavily on both the Harris measure and a Gaussian scale space representation. The principle of Harris corner detector algorithm is as at a corner, the image intensity will change largely in multiple directions. By sifting local window, this can be formulated by examining the changes of intensity. When the window is shifted in an arbitrary direction around a corner point, the image intensity will change greatly. The Harris detector uses the second moment matrix as the basis of its corner decisions. It is called the autocorrelation matrix. This matrix has values which are closely related to the derivatives of image intensity

The method first builds up two separate scale spaces for the Harris function and the Laplacian. Harris function on each scale level is used to localize aspirant points and Laplacian is then attains those points for an extreme over scales. The resulting points are robust to changes in image rotation, scale, illumination, and noise.

The Harris function is defined by,

$$
\begin{gathered}
u\left(X, \sigma_{i}, \sigma_{D}\right)= \\
\sigma_{D}{ }^{2} g\left(\sigma_{i}\right) *\left[\begin{array}{ll}
L_{x}{ }^{2}\left(X, \sigma_{D}\right) & L_{x} L_{y}\left(X, \sigma_{D}\right) \\
L_{x} L_{y}\left(X, \sigma_{D}\right) & L_{x}{ }^{2}\left(X, \sigma_{D}\right)
\end{array}\right]
\end{gathered}
$$

In the above equation,

$\sigma_{1}$ - Integration Scale

$\sigma_{D}$ - Differentiation scale

$L_{x}$ - Derivative computed in $\mathrm{x}$ direction

$\mathrm{L}(\mathrm{X}, \sigma)$ - the convolution result of $\mathrm{g}(\sigma)$ and image

$\mathrm{I}(\mathrm{X})$.

$$
\begin{aligned}
& \mathrm{L}(\mathrm{X}, \sigma)=\mathrm{g}(\sigma) * \mathrm{I}(\mathrm{X}) \text {. ----- (2) } \\
& \mathrm{g}(\sigma)-\text { Gaussian kernel function with scale } \sigma \\
& \mathrm{g}(\sigma)=\frac{1}{2 \pi \sigma^{2}} e^{-X^{T} X / 2 \sigma^{2}}
\end{aligned}
$$

Feature Detection is done as follows

1. Extracts interest points at multiple scale levels which are the local spatial maxima of the scale-adapted Harris function. a) At each level of scale space we detect interest points by detecting the local maxima in the image plane

b) Find local maxima greater than threshold

c) For each of the candidate point found at each levels, verify if it forms a maximum in the scale direction

2. It then uses the Laplacian for scale selection and verify for each of the initial points whether the LoG attains a maximum at the scale of the point
Scale selection determines the characteristic scale of a local structure, i.e. the scale with maximum similarity between the feature detection operator and the local image structures.

This scale is determined as the extreme over scale of a given function and is independent of the image resolution. Corresponding to different local structures centered at one point, there might be several extrema. The Laplacian operator is used for scale selection since it gave the best results in our experimental comparison. Initial multi-scale interest points are rejected if the Laplacian attains no extremum at the scale of extraction or if the Laplacian response is below a given threshold. We then obtain a set of scale-invariant points with associated scales.

Interest points, detected for the same image structure, change their location relative to the detection scale in the gradient direction.

Harris-Laplacian detector typically returns a much smaller number of points than the Laplacian or DoG detectors. It selects a complementary type of regions: corners and regions of "high information content".

\subsection{The SUSAN detector: $[10,13,14]$}

SUSAN stands for Smallest Univalue Segment Assimilating Nucleus. This method is use the gradient differential of image directly and It is a generic low-level image processing technique, It is used for corner detection as well as edge detection and noise suppression. The algorithm is proposed by Smith and Brady is based on brightness comparisons within a circular mask. SUSAN assumes that within a relatively small circular region pixels belonging to a given object will have relatively uniform brightness. The algorithm computes the number of pixels with similar brightness to the pixel at the center of the mask. The center pixel will be referred to as the nucleus of the mask and the area with similar intensity to the nucleus, the USAN (Univalue Segment Assimilating Nucleus) area. The USAN area is maximum within the homogeneous area but falls to a minimum at an edge and to an even smaller value corresponding to a local minimum at a corner

It finds places where individual region boundaries have high curvature by analyzing different regions separately, using direct local measurements, i.e., finds corners formed by single regions. If the junctions involving more than two regions for example At ' $\mathrm{T}$ ' junctions more than one region may contribute to the detection of a "corner, All the regions are correctly processed and corner is decided.

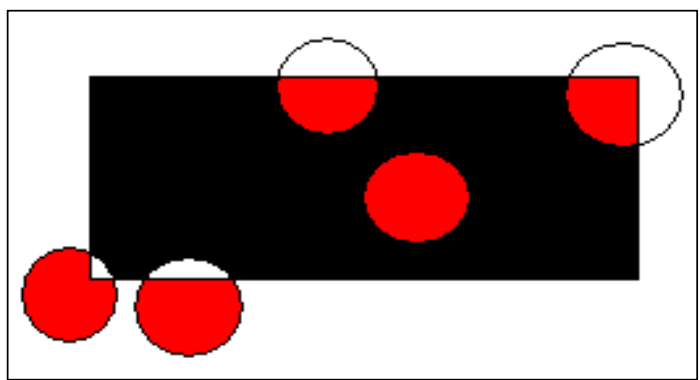

Figure 3.1 Circular mask applied to different positions of a black rectangle with Red colored USAN. (USAN becomes smaller as it approaches an edge and this reduction is stronger at corners)

Algorithm for Susan as per [10]

1. For each pixel of an image a circular mask, of minimum radius around a centre point (nucleus) is taken.

2. Calculate the difference in brightness between each pixel of the mask and that of its nucleus 
3. Addition of similar intensity levels to that of the nucleus is done within the circular mask

4. Corner response is calculated by getting difference after comparing the brightness calculated with the geometric threshold, which is usually half of the biggest of the numbers calculated.

5. At the proper corner the SUSAN area corners will have centers of gravity sufficiently far from the nucleus of the mask At the proper corner, the forming part of the USAN area are those pixels falling on a straight line from the nucleus of the mask to its center of gravity, so the remaining areas of the mask will only contain false corners and can be discarded.

5. The initial corner response image is suppressed so that local maxima are searched for in smaller pixel regions.

Because it is not derived from differential geometry, the SUSAN corner finder performs very well even in presence of noise.

\section{4 Gilles [11]}

For matching two images, Gilles (Gilles, 1998), investigates salient local image patches. Visual Saliency is the perceptual quality that makes a region or object stand out relative to its neighbors and so it captures our attention. Silent features are generally determined from the local differential structure of images. Gilles introduced a set of salient features based on a local entropy analysis of an image. Gross global transforms do not affect the saliency of the features, if it is defined locally.

Gilles defines saliency in terms of local signal, That is he suggests the use of Shannon entropy of local attributes. Higher entropy is found in areas corresponding to high signal complexity tend to have flatter distributions. It is the high complexity of a suitable descriptor that can be used as a measure of local saliency.

Given a point $\mathrm{x}$, a local neighborhood $\mathrm{R}_{\mathrm{X}}$, and a descriptor $\mathrm{D}$ that takes on values $\left(\mathrm{d}_{1} ;::: ; \mathrm{d}_{\mathrm{r}}\right)(\mathrm{e}$.g. $\mathrm{D}$ would range from 0 to 255 in an 8 bit grey level image), local entropy is defined as:

$H_{D}, R_{x}=-\sum P_{D} R_{x}\left(d_{i}\right) \log _{2} P_{D}, R_{x}\left(d_{i}\right)$

Where $\mathrm{P}_{\mathrm{D}} ; \mathrm{Rx}(\mathrm{di})$ is the probability of descriptor $\mathrm{D}$ taking the value di in the local region $\mathrm{Rx}$.

Complexity in real images is rare is the underlying assumption. This is not true in the case of noise or self-similar images where complexity is independent of scale and position. In paper [11] they have discussed the idea of visual saliency which suggests that certain regions of the image are better than others at describing content. In this paper we have taken Gilles method for feature extraction

Steps to follow for feature extraction using Gilles method 1. Circular averaging filter (pillbox) within the square matrix of side $2 *$ radius +1 is created. Radius is selected as per user choice and default value is 5 .

2; Local entropy is calculated using filter calculated as above and the image intensity

3. Find Local maxima

4. Check the points and Keep the points above the threshold value

The algorithm is sensitive to small changes and noise in the image. The positions of the icons rarely remain stable over time on a salient feature, they oscillate around it

\section{WATERMARK EMBEDDING AND DETECTION}

Watermark embedding process:
1. Select original image
2. Extract feature points of the image
3. Find non overlapping regions
4. Apply attacks and check feature survival
5. Select regions using pruning algorithm
6. Apply DCT to embed watermark in region

Watermark detection Process:

1. Get the received image.
2. Extract features
3. Find non overlapping regions
4. Extract watermark from region
5. Compare original watermark with extracted
watermark for detection decision.

\section{The Discrete Cosine Transform (DCT) Technique}

Another watermarking technique is based on a direct cosine transformation (DCT)[5] transformation. The DCT algorithm is one of the main components of the JPEG compression technique.

DCT transformation separates the image into spectral sub-bands depending on the importance with respect to the image's visual quality. It can separate the Image into High, Middle and Low Frequency components. For most images, much of the signal energy lies at low frequencies. These appear in the upper left corner of the DCT, so the modification of low frequency can be catch by human eyes. Modification of high frequencies can cause local distortion along with edges. Middle frequencies modification can not affect the image quality so transform coefficients as thus set threshold value in this area.

The one dimensional DCT is defined as

$$
C(u)=\alpha(u) \sum_{x=0}^{N-1} f(x) \cos \left[\frac{\pi(2 x+1) u}{2 N}\right]
$$

The two dimensional DCT is defined a

$$
\begin{gathered}
C(u, v) \\
=\alpha(u) \alpha(v) \sum_{x=0}^{N-1} \sum_{y=0}^{N-1} f(x, y) \cos \left[\frac{\pi(2 x+1) u}{2 N}\right] \cos \left[\frac{\pi(2 y+1) v}{2 N}\right] \\
\alpha(u)= \begin{cases}\sqrt{\frac{1}{N}}, & \text { for } u=0 \\
\sqrt{\frac{2}{N}}, & \text { for } u \neq 0\end{cases}
\end{gathered}
$$

Inverse DCT is

$$
\begin{aligned}
& f(x, y) \\
& =\sum_{u=0}^{N-1} \sum_{v=0}^{N-1} \alpha(u) \alpha(v) C(u, v) \cos \left[\frac{\pi(2 x+1) u}{2 N}\right] \cos \left[\frac{\pi(2 y+1) v}{2 N}\right]
\end{aligned}
$$

Where $\mathrm{u}=1,2, \ldots . \mathrm{N}-1$ and $\mathrm{v}=1,2, \ldots \ldots \mathrm{M}-1$ 
DCT is faster and can be implemented in $O(n \log n)$ operations.

Watermark Embedding Process as [5, 6]:

The embedding watermark is as follows.

1. Sequentially extract out every 8-bit data from watermarkbit-stream.

2. Obtain a random number, generated by pseudo random system, which points to one of n blocks of host image.

3. Embed extracted the 8-bit watermarking data into the 8 lower-band coefficients in the block pointed by previous step.

4. Repeat step 1 to step 3, until the watermark bit stream is run out.

5. The proposed that replace bit to embedded watermark bit stream, and it was hidden at position bit 4 in the selected 8-bit coefficient. If the watermark bit is " 1 " then bit 4 to " 1 " otherwise " 0 ".

Extracting Watermarked Image [5, 6]:

1) Perform DCT transform on watermarked image and original host image.

2) Substract original host image from watermarked image.

3) Multiply extracted watermark by scaling factor to display.

\section{Advantages}

- DCT domain watermarking is comparatively much better than the spatial domain encoding since DCT domain watermarking can survive against the attacks such as noising, compression, sharpening, and filtering.

- It use JPEG compression method to apply DCT watermarking as a parameter. One may use different parameters related to image processing, and these parameters might provide equal or even stronger robustness against various attacks based on image processing.

- Discrete cosine transform (DCT), where pseudorandom sequences, such as M sequences, are added to the DCT at the middle frequencies as signatures

\section{EXPERIMENTAL RESULTS}

Well known Lena and Baboon images of size $512 \times 512$ (JPEG/BMP images) are taken for experimentation.

In all feature extraction methods we get regions of different size. We have taken first 20 non overlapping regions(If number of regions are more than 20) having greater sizes in Harris laplace and LoG method. In Gilles and Susan we kept size of radius 20 each and first 20 regions are taken for comparison.

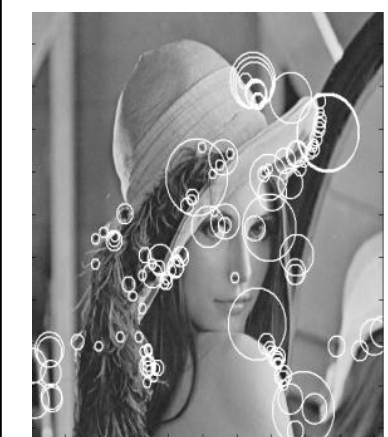

Figure 5.1 Extracted regions By LoG

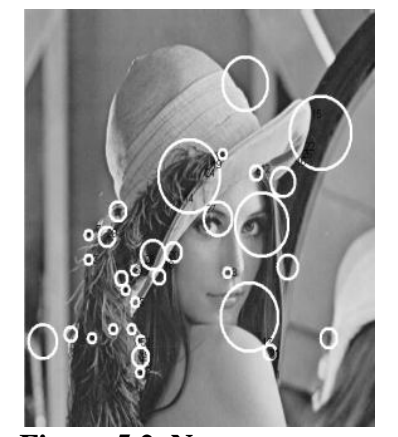

Figure 5.2 Non overlapping regions

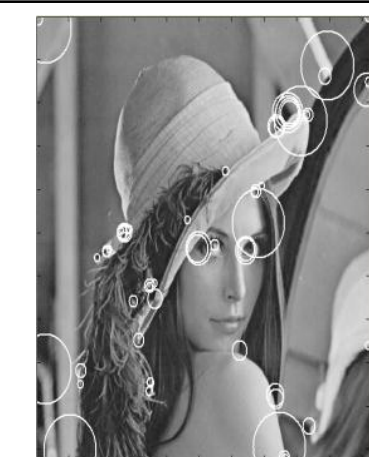

Figure 5.3 Extracted regions by Harris Laplace

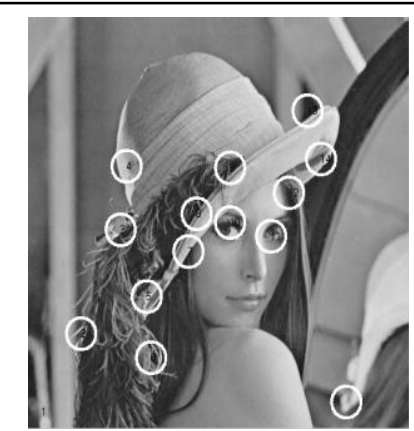

Figure 5.5 Non overlapping regions by Gilles method

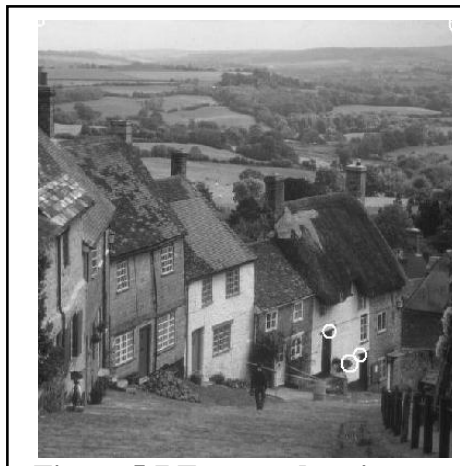

Figure 5.7 Extracted regions by Harris Laplace

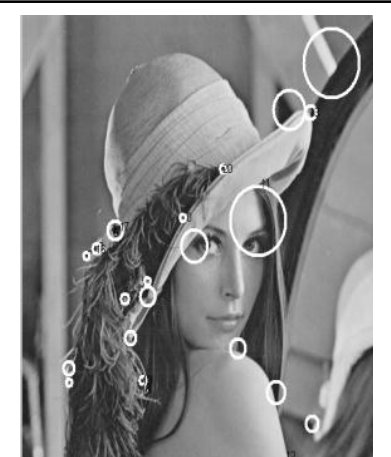

Figure 5.4 Non overlapping regions

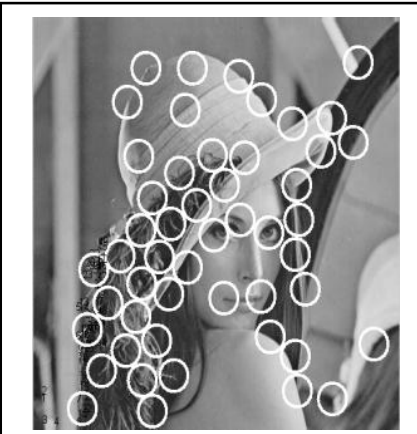

Figure 5.6 Non overlapping regions by Susan

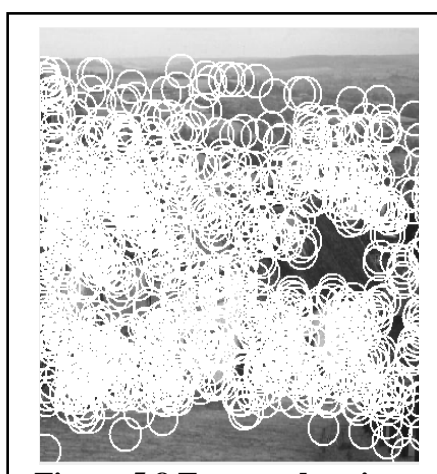

Figure 5.8 Extracted regions by Susan

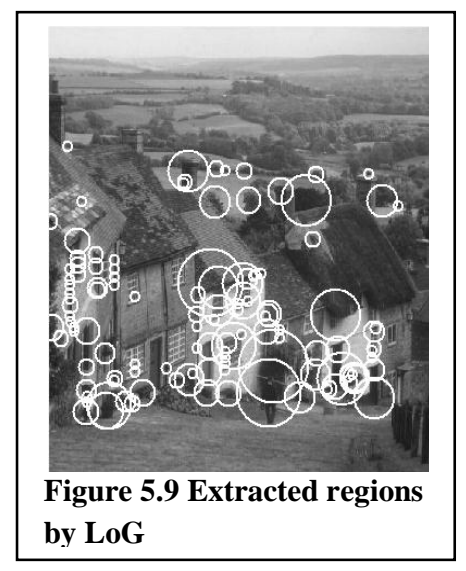


After extraction of feature regions, non overlapping regions are found and then each region is saved and applied to different attacks and after attacks are applied survival of each region is checked using PSNR value and table is generated. The region is selected for watermark embedding which is survived with more number of attacks.

Number of regions and survival of region also compared with different feature extraction methods.

\begin{tabular}{|c|c|c|c|c|c|c|c|c|}
\hline 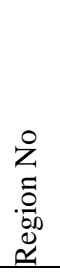 & $\begin{array}{l}0 \\
.0 \\
0 \\
z \\
0 \\
0 \\
0 \\
0 \\
0\end{array}$ & 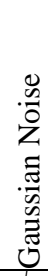 & 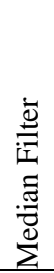 & $\frac{1}{2}$ & 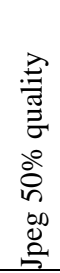 & 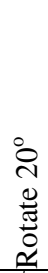 & $\Xi$ & 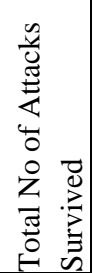 \\
\hline 1 & $\sqrt{ }$ & $\sqrt{ }$ & $\mathrm{x}$ & $\sqrt{ }$ & $\mathrm{x}$ & $\sqrt{ }$ & $\sqrt{ }$ & 5 \\
\hline 2 & $\sqrt{ }$ & $\sqrt{ }$ & $\mathrm{X}$ & $\sqrt{ }$ & $\mathrm{x}$ & $\sqrt{ }$ & $\sqrt{ }$ & 5 \\
\hline 3 & $\mathrm{x}$ & $\sqrt{ }$ & $\mathrm{x}$ & $\sqrt{ }$ & $\mathrm{x}$ & $\sqrt{ }$ & $\mathrm{x}$ & 3 \\
\hline 4 & $\mathrm{x}$ & $\sqrt{ }$ & $\mathrm{x}$ & $\sqrt{ }$ & $\mathrm{x}$ & $\sqrt{ }$ & $\sqrt{ }$ & 4 \\
\hline 5 & $\mathrm{x}$ & $\mathrm{x}$ & $\mathrm{x}$ & $\sqrt{ }$ & $\mathrm{x}$ & $\sqrt{ }$ & $\sqrt{ }$ & 3 \\
\hline 6 & $\mathrm{x}$ & $\mathrm{x}$ & $\mathrm{x}$ & $\sqrt{ }$ & $\mathrm{x}$ & $\sqrt{ }$ & $\mathrm{x}$ & 2 \\
\hline 7 & $x$ & $\mathrm{x}$ & $\mathrm{x}$ & $\sqrt{ }$ & $\mathrm{x}$ & $\sqrt{ }$ & $\sqrt{ }$ & 3 \\
\hline 8 & $\mathrm{x}$ & $\mathrm{x}$ & $\mathrm{x}$ & $\sqrt{ }$ & $\mathrm{x}$ & $\sqrt{ }$ & $\sqrt{ }$ & 3 \\
\hline 9 & $\mathrm{x}$ & $\mathrm{x}$ & $\mathrm{x}$ & $\sqrt{ }$ & $\mathrm{x}$ & $\sqrt{ }$ & $\mathrm{x}$ & 2 \\
\hline 10 & $\mathrm{x}$ & $\mathrm{x}$ & $\mathrm{x}$ & $\sqrt{ }$ & $\mathrm{x}$ & $\sqrt{ }$ & $\sqrt{ }$ & 3 \\
\hline 11 & $\mathrm{x}$ & $\mathrm{x}$ & $\mathrm{x}$ & $\sqrt{ }$ & $\mathrm{x}$ & $\sqrt{ }$ & $\sqrt{ }$ & 3 \\
\hline 12 & $\mathrm{x}$ & $\mathrm{x}$ & $\mathrm{x}$ & $\sqrt{ }$ & $\mathrm{x}$ & $\sqrt{ }$ & $\sqrt{ }$ & 3 \\
\hline 13 & $\mathrm{x}$ & $\mathrm{x}$ & $\mathrm{x}$ & $\sqrt{ }$ & $\mathrm{x}$ & $\mathrm{x}$ & $\mathrm{x}$ & 1 \\
\hline 14 & $\mathrm{x}$ & $x$ & $\mathrm{x}$ & $\sqrt{ }$ & $\mathrm{x}$ & $\sqrt{ }$ & $\mathrm{x}$ & 2 \\
\hline 15 & $\mathrm{x}$ & $\mathrm{X}$ & $\mathrm{x}$ & $\sqrt{ }$ & $\mathrm{x}$ & $\mathrm{x}$ & $\mathrm{x}$ & 1 \\
\hline 16 & $\mathrm{x}$ & $\mathrm{x}$ & $\mathrm{x}$ & $\sqrt{ }$ & $\mathrm{x}$ & $\mathrm{x}$ & $\mathrm{x}$ & 1 \\
\hline 17 & $\mathrm{x}$ & $\mathrm{x}$ & $\mathrm{x}$ & $\sqrt{ }$ & $\mathrm{x}$ & $x$ & $\mathrm{x}$ & 1 \\
\hline 18 & $\mathrm{x}$ & $x$ & $x$ & $\sqrt{ }$ & $\mathrm{x}$ & $x$ & $x$ & 1 \\
\hline 19 & $x$ & $\mathrm{x}$ & $\mathrm{x}$ & $\sqrt{ }$ & $\mathrm{x}$ & $\mathrm{x}$ & $\mathrm{x}$ & 1 \\
\hline
\end{tabular}

Table 1. Lena image Regions survived for different attacks(Regions extracted by Harris-Laplace feature extraction method )

Similar to this other tables are generated for each feature extraction methods. As above table table for each extraction method is generated and final comparison table 2 is generated.

\begin{tabular}{|c|c|c|c|c|c|c|c|}
\hline & $\begin{array}{l}0 \\
.0 \\
0 \\
z \\
\tilde{0} \\
0 \\
0 \\
0 \\
0\end{array}$ & 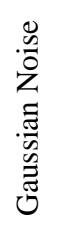 & 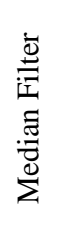 & $\frac{\pi}{2}$ & 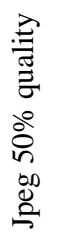 & 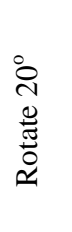 & 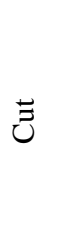 \\
\hline Gilles & $0 / 13$ & $13 / 13$ & $0 / 13$ & $13 / 13$ & $0 / 13$ & $13 / 1$ & $13 / 1$ \\
\hline LoG & $5 / 31$ & $10 / 31$ & $0 / 31$ & $31 / 31$ & $0 / 31$ & $13 / 1$ & $13 / 1$ \\
\hline Harri & $2 / 19$ & $4 / 19$ & $0 / 19$ & $19 / 19$ & $0 / 19$ & $13 / 1$ & $9 / 19$ \\
\hline Susan & $0 / 49$ & $49 / 49$ & $0 / 49$ & $49 / 49$ & $0 / 49$ & $49 / 4$ & $49 / 4$ \\
\hline
\end{tabular}

Table 2 Lena image Regions survived for different attacks (Regions extracted by Gilles method, there are 13 regions and in LoG there are 31 regions) or each attack the number of regions survived / Total number of Regions are displayed are displayed

For Rotation attack is tested for different angles for testing rotation invariance and same results are found for LoG , Susan and Gilles method that all regions have feature survival for rotation attack for 20 to $100^{\circ}$

After Scaling by $75 \%$ also almost same results are found in LoG, Susan, Harris Laplace and Gilles method for all regions.

\section{Watermark embedding and Extraction results :}

Watermark embedded in regions having maximum feature survival (resistant to maximum number of attacks) using DCT technique and extracted successfully. Only small regions are not selected for embedding the watermark.

\section{CONCLUSION AND FUTURE WORK}

As per research work done for robustness of watermarking, there are many techniques are suggested as spatial domain and frequency domain based watermarking techniques. Watermarking in frequency domain as DFT, DCT, DWT are more robust than watermarking in spatial domain because information can be spread out to entire image.

As features of the image have high invariance to distortions, they can be used as a key to find the insertion location. The goal is to resist both geometric distortion and signal processing attacks, feature based watermarking scheme is suggested in combination with frequency or spatial domain based watermarking. Since no watermarking algorithm resists all the attacks. Still we can find better which will give more robust watermark.

Feature extraction can be done using different methods such as Harris Laplacian, Hessian-Affine detector, MSER, IBR, Susan, Laplacian-of-Gaussian (LoG), Gilles etc. Each method gives different results. As compared to LoG, Harris laplacian and Gilles gives less regions. Susan gives more number of regions. LoG and Harris laplacian are good for RST attacks. As per [12] the SUSAN detector is efficient and it avoids computation of derivatives, however the absence of smoothing makes it more susceptible to noise and it is gives rotation invariant features. By experimental results Susan is more efficient and gives more number of regions. In the scale-invariant group Harris-Laplace shows high repeatability and localization accuracy inherited from the Harris detector but gives less regions as shown in figure 5.7.In that case we have to select other method such as LoG or 
Susan shown in figure 5.8 and 5.9. Pruning also gives better results for selecting feature region sets.

Future work can be done for selecting different robust features and selecting proper embedding technique can improve the robustness of watermark and different Optimization techniques can be used for selecting different regions further for watermark embedding.

\section{ACKNOWLEDGEMENT}

Our thanks to the experts Mr. Vincent Garcia for providing matlab code for feature extraction and Mr. Joaquim Luis 'Susan' algorithm for research purpose at site id http://www.mathworks.com/matlabcentral/fileexchange/1789

\section{4-keypoint-extraction/content/}

\section{REFERENCES}

[1] liu quan, jiang xuemei "researches on uniform meaningful watermark?, proceedings of the $20026 \mathrm{t}$ international conference on signal processing, 2002.

[2] S. Voloshynovskiy, S. Pereira, T. Pun, University of Geneva J.J. Eggers and J.K. Su, University of ErlangenNuremberg, Attacks on Digital Watermarks: Classification, Estimation-based Attacks and Benchmarks

[3] Fabien A.P. Petitcolas, Ross J. Anderson, and Markus G. Kuhn "Attacks on Copyright Marking Systems"

[4] STIRMARK bench mark http://www.petitcolas.net/fabien/watermarking/stirmark/

[5] Mrs Neeta Deshpande, Dr. Archana rajurkar, Dr. R. manthalkar Review of Robust Video Watermarking algorithms, (IJCSIS) International Journal of Computer Science and Information Security, Vol. 7, No. 3, March 2010.
[6] K. R. Rao and P. Yip, "Discrete Cosine Transform: Properties, Algorithms, Advantages, Applications"

[7] Chih-Wei Tang and Hsueh-Ming Hang, "A FeatureBased Robust Digital Image Watermarking Scheme", IEEE Transactions on signal processing vol 51, NO. 4, APRIL 2003

[8] Jen-Sheng Tsai, Win-Bin Huang, Chao-Lieh Chen, YauHwang Kuo, "A Feature-Based Digital Image Watermarking For Copyright Protection And Content Authentication "Image Processing,IEEE International Conference 2007

[9] Jen-Sheng Tsai, Win-Bin Huang, and Yau-Hwang Kuo, "On the Selection of Optimal Feature Region Set for Robust Digital Image Watermarking" IEEE Transactions On Image Processing, Vol. 20, No. 3, March 2011

[10] B. J. Lei, Emile A. Hendriks, M.J.T. Reinders "On Fea ture Ex traction from Images" 1999

[11] [11] Timor Kadir, Michael BradySaliency, "Scale and Image Description"

[12] Tinne Tuytelaars and Krystian Mikolajczyk "Local Invariant Feature Detectors: A Survey"

[13] Kristen Grauman and Bastain Leibe "Local Features: Detection and Description"

[14] Stephen m. smith, j. michael brady, "SUSAN-A New Approach to Low Level Image Processing"

[15] Xiang-Yang Wang1;2; Jun Wu1A Feature-based Robust Digital Image Watermarking Against Desynchronization Attacks 